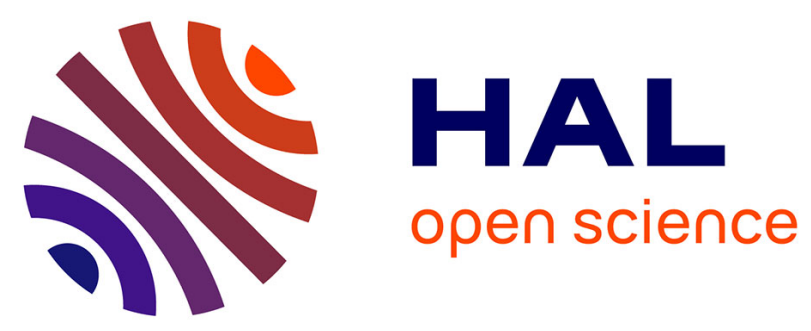

\title{
Phase measurement of soft x-ray multilayer mirrors
}

Sébastien de Rossi, Charles Bourassin-Bouchet, Evgueni Meltchakov, Angelo Giglia, Stefano Nannarone, Franck Delmotte

\section{To cite this version:}

Sébastien de Rossi, Charles Bourassin-Bouchet, Evgueni Meltchakov, Angelo Giglia, Stefano Nannarone, et al. Phase measurement of soft x-ray multilayer mirrors. Optics Letters, 2015, 40 (19), pp.4412-4415. 10.1364/OL.40.004412 . hal-01202422

\section{HAL Id: hal-01202422 \\ https://hal-iogs.archives-ouvertes.fr/hal-01202422}

Submitted on 30 Sep 2015

HAL is a multi-disciplinary open access archive for the deposit and dissemination of scientific research documents, whether they are published or not. The documents may come from teaching and research institutions in France or abroad, or from public or private research centers.
L'archive ouverte pluridisciplinaire HAL, est destinée au dépôt et à la diffusion de documents scientifiques de niveau recherche, publiés ou non, émanant des établissements d'enseignement et de recherche français ou étrangers, des laboratoires publics ou privés. 


\title{
Phase measurement of soft x-ray multilayer
} mirrors

\author{
Sébastien de Rossi, ${ }^{1, *}$ Charles Bourassin-Bouchet, ${ }^{1,2}$ Evgueni Meltchakov, ${ }^{1}$ \\ Angelo Giglia, ${ }^{3}$ Stefano Nannarone, ${ }^{3}$ and Franck Delmotte ${ }^{1}$ \\ 'Laboratoire Charles Fabry, UMR 8501, Institut d'Optique, CNRS, Univ. Paris Sud 11, 91127 Palaiseau, France \\ ${ }^{2}$ Synchrotron SOLEIL, Saint Aubin, BP 34, 91192 Gif-sur-Yvette, France \\ ${ }^{3}$ Istituto Officina dei Materiali-Consiglio Nazionale delle Ricerche Laboratorio Tecnologie, Avanzate e NanoSCienza, Area Science Park Basovizza, \\ S.S. $14 \mathrm{Km}$ 163.5, 34149 Trieste, Italy \\ *Corresponding author: sebastien.derossi@institutoptique.fr
}

Received 1 July 2015; revised 28 August 2015; accepted 31 August 2015; posted 1 September 2015 (Doc. ID 244009); published 00 MONTH 0000

\begin{abstract}
We propose a new model enabling the extraction of the phase of a multilayer mirror from photocurrent measurements in the soft $x$ rays. In this range, the effects of the mean free path of the electrons inside the stack can no longer be neglected, which prevents the phase reconstruction by conventional photocurrent measurements. The new model takes into account this phenomenon and thus extends up to the $x$ rays the applicability range of the technique. This approach has been validated through a numerical and experimental study of chromium/scandium multilayers used near $360 \mathrm{eV}$. To our knowledge, this work constitutes the first measurement of the phase of a multilayer mirror in the soft x-ray range. () 2015 Optical Society of America
\end{abstract}

OCIS codes: (120.5050) Phase measurement; (340.7470) X-ray mirrors; (230.4170) Multilayers.

http://dx.doi.org/10.1364/OL.99.099999

Multilayer mirrors have proven to be promising candidates for transporting and shaping attosecond pulses in the extreme ultraviolet and $\mathrm{x}$ rays (XUV) [1-3]. As opposed to conventional XUV multilayer mirrors, attosecond multilayer optics must be characterized both in reflectivity and phase to ensure an efficient reflection of the light pulses. Phase characterization of the mirror can be performed through the measurement of the reflected attosecond pulse [2,3]. A powerful alternative consists of measuring the surface photocurrent, that is, the amount of electrons emitted at the surface of the stack under irradiation. Indeed, the formation of a standing wave inside the multilayer under XUV illumination naturally relates the surface photocurrent to the phase of the mirror [4]. This approach has been successfully used in the past to measure the phase of multilayers below $120 \mathrm{eV}$ [5-8]. However, extending the photocurrent technique to higher energies has remained challenging. Indeed, one necessary condition to recover the phase from the photocurrent is that the collected electrons only originate from the surface of the stack. For higher photon energies, however, the electron mean free path (MFP) in the material becomes comparable to the period of the optical field inside the multilayer, which prevents a direct phase extraction. This limitation is so unfortunate that strong efforts have been made to extend attosecond sources to higher energies, with spectra already observed in the water window $(280-530 \mathrm{eV})$ [9]. Transporting and tayloring such pulses with multilayer mirrors thus requires an adaptation of phase metrology techniques to this spectral range.

In this Letter, we propose a model for photocurrent measurements giving access to the phase of a multilayer mirror even when the effects of the electron MFP cannot be neglected. We demonstrate the approach experimentally by measuring the phase of two periodic chromium/scandium multilayer stacks designed to reflect light near $360 \mathrm{eV}$. An estimate of the electron MFP in the surface layer is also deduced from these measurements. To our knowledge, this constitutes the first measurement of the phase of a multilayer mirror in the water-window spectral range.

In a multilayer stack under irradiation, a standing wave appears due to the superposition of the incident and reflected waves, the amplitudes of which are, respectively, denoted $A_{i}(\omega, z)$ and $A_{r}(\omega, z)$, with $\omega$ being the angular frequency and $z$ being the depth in the stack, the surface being at $z=0$ [see Figs. 1(a) and 1(b)]. In that case, the photocurrent $i(\omega)$ measured at the surface of the sample with a picoammeter reads

$$
i(\omega)=\int_{-\infty}^{0} e^{-z / \alpha} C(\omega, z)\left|A_{i}(\omega, z)+A_{r}(\omega, z)\right|^{2} \mathrm{~d} z,
$$

where $\alpha$ represents the MFP and $C(\omega, z)$ is the electron yield of the material, that is, its ability to create photoelectrons $[4,10]$. Equation (1) gives a rigorous expression for the photocurrent but does not relate it to the phase of the stack. Therefore, in the conventional approach, one assumes that the MFP is sufficiently small compared to the variations of the standing wave, that is, that all the electrons detected at the surface see a standing wave reading as $C(\omega, 0)\left|A_{i}(\omega, 0)+A_{r}(\omega, 0)\right|^{2}$. When compared to a reference current $i_{\text {ref }}$ obtained from a sample made of the same surface material as the stack under test 
(i.e., the same $c$ coefficient) and having a negligible reflectivity, the normalized photocurrent $\eta$ is obtained [5-8]:

$$
\eta=\frac{i(\omega)}{i_{\mathrm{ref}}(\omega)} \approx 1+R(\omega)+2 \sqrt{R(\omega)} \cdot \cos \phi_{\mathrm{mir}}(\omega),
$$

where $R=\left|A_{r} / A_{i}\right|^{2}(\omega, z=0)$ and $\phi_{\text {mir }}=\varphi_{r}-\varphi_{i}$ correspond, respectively, to the reflectivity and phase of the multilayer stack. Consequently, the latter phase can be straightforwardly obtained by expressing $\phi_{\text {mir }}(\omega)$ as a function of $\eta(\omega)$ and $R(\omega)$, which are two measurable quantities.

This formalism works well for photon energies below $100 \mathrm{eV}$. However, as the period of the standing wave decreases with the wavelength, thickness of layers becomes of the order of magnitude of the MFP (a few nanometers) [11]. Therefore, the electrons experience a standing wave that is no longer constant with $z$ before they reach the surface. In the model proposed here, we instead consider that the standing wave near the surface locally takes the form of an oscillatory field of constant frequency and amplitude. The input and output waves $A_{i, r}(\omega, z)$ now read $\left|A_{i, r}(\omega, 0)\right| \cdot \exp \left[i\left(\varphi_{i, r}(\omega, 0) \pm k z-\omega t\right)\right]$, with $k=k^{\prime}+i k^{\prime \prime}=\frac{\omega}{c}\left[n^{\prime}+i n^{\prime \prime}\right] \cos \theta, \theta$ being the angle of incidence of the beam on the mirror. The term $c$ is now considered to be independent of $z$, which can be fulfilled by depositing a top layer thicker than $\alpha$ or by assuming that $C$ is the same for all materials. Inserting these expressions into Eq. (1) and using the slowly varying envelope approximation, one can factorize out of the integral the terms varying slowly with $z \cdot \eta$ now reads $1+R+2 \sqrt{R} \int e^{-\left(\alpha^{-1}+2 k^{\prime \prime}\right) z} \cos \left(\phi_{\text {mir }}+2 k^{\prime} z\right) \mathrm{d} z$. Finally, using trigonometric relations, one can rewrite it in the compact form:

$$
\eta=1+R+2 \sqrt{R} \cdot \frac{\cos \phi_{\mathrm{mir}}-\gamma \sin \phi_{\mathrm{mir}}}{1+\gamma^{2}},
$$

with $\gamma=2 k^{\prime} /\left(\alpha^{-1}+2 k^{\prime \prime}\right)$. If assuming that the penetration depth of the XUV radiation in the material is much larger than the MFP $\left(k^{\prime \prime} \ll \alpha^{-1}\right), \gamma$ simplifies into $2 k^{\prime} \alpha$. For photon energies $\lesssim 100 \mathrm{eV}$, the period of the standing wave is sufficiently large so that $\gamma \ll 1$ and $\eta$ takes the form given in Eq. (2), whereas $\gamma \approx 1$ for higher energies.

In order to validate this model, we perform numerical simulations shown in Figs. 1(b) and 1(c). We use a homemade matlab code based on the iterative approach using the optical constants from the CXRO database. The chosen mirror is a chromium/scandium $(\mathrm{Cr} / \mathrm{Sc})$ multilayer stack $(20$ periods of $1.2 \mathrm{~nm}$ of $\mathrm{Cr} / 1.2 \mathrm{~nm}$ of Sc, capping layer of $1.9 \mathrm{~nm}$ of $\mathrm{Si}$ $+1.5 \mathrm{~nm}$ of $\mathrm{SiO}_{2}$ ) at $45^{\circ}$ incidence angle). In these conditions, the standing wave depicted in Fig. 1(b) is obtained inside the stack. We then calculate the normalized photocurrent for various MFP using (i) the rigorous photocurrent integration, that is, Eq. (1) assuming the $c$ coefficient constant with $z$; (ii) the simplified model assuming a zero MFP described by Eq. (2); or (iii) the new model described by Eq. (3). According to the results in Fig. 1(c), for $\alpha=0 \mathrm{~nm}$, the three models agree perfectly. Interestingly, slightly increasing the MFP up to $0.1 \mathrm{~nm}$ induces a visible shift of the integrated photocurrent that is well reproduced by the new model. It is remarkable to see that even a small MFP can create significant deviations from the simplified model. When increasing the MFP further, the characteristic oscillations of the photocurrent undergo a phase shift close to $\pi / 2$, as well as an attenuation. Once again, these features are perfectly retrieved by the new

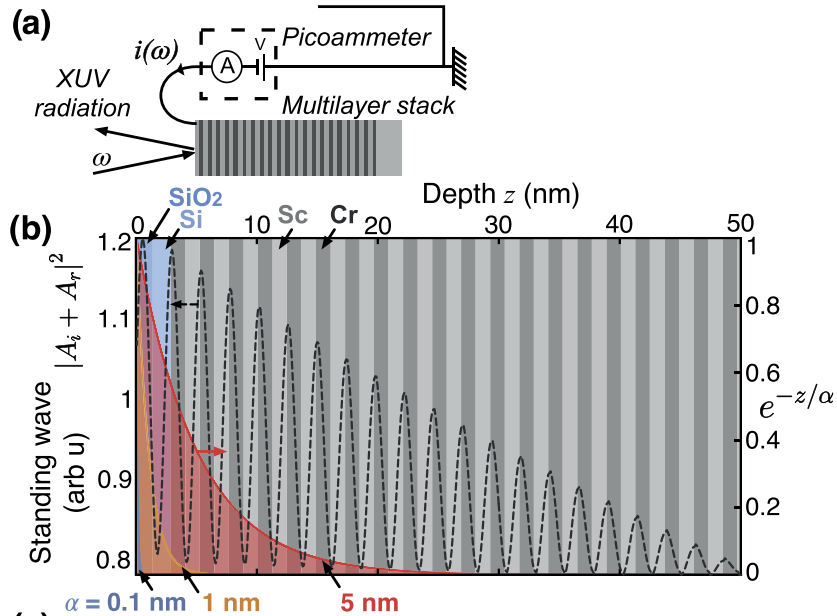

(c)

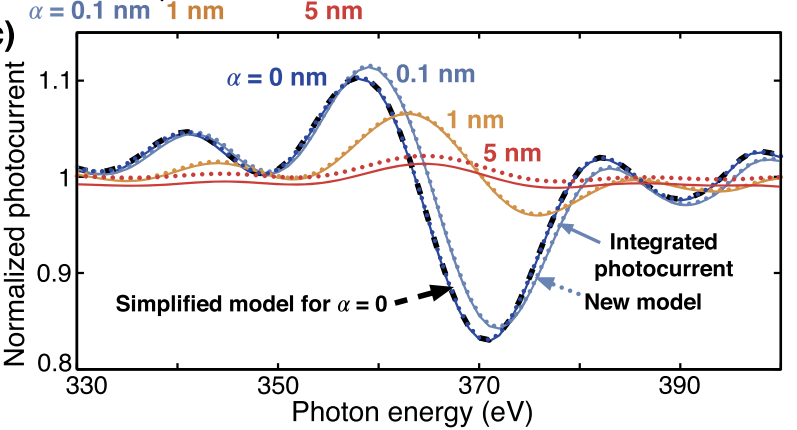

Fig. 1. (a) Principle of a surface photocurrent measurement. (b) Evolution of the standing wave inside the stack (dashed line) compared to the range of influence of various MFP (shaded curves). (c) Normalized photocurrents $\eta(\omega)$ obtained with the rigorous integrated model described by Eq. (1) (continuous lines) and with the new model described by Eq. (3) (dotted lines) for various MFP. The photocurrent obtained with the simplified model [Eq. (2)] is also given for $\alpha=0 \mathrm{~nm}$ (dashed line).

model. These observations can be explained by Eq. (3). When increasing $\alpha$, the term $\gamma \sin \phi_{\text {mir }}$ tends to dominate $\cos \phi_{\text {mir }}$ and thus to phase shift $\eta$ by $\pi / 2$. At the same time, the term $1 /\left(1+\gamma^{2}\right)$ induces an overall attenuation of the signal. For MFP $\geq 5 \mathrm{~nm}$, the integrated photocurrent is even more attenuated, but the new model becomes unable to fully reproduce it, indicating that the slowly varying envelope approximation is no longer valid.

The main advantage of the new model compared to the rigorous photocurrent integration is that Eq. (3) can be inverted in order to express $\phi_{\text {mir }}(\omega)$ as a function of $R, \gamma$, and $\eta$, leading to

$$
\phi_{\text {mir }}(\omega)=\arccos \left[A+\gamma \sqrt{\frac{1}{1+\gamma^{2}}-A^{2}}\right],
$$

with $A$ equal to $\frac{\eta-1-R}{2 \sqrt{R}}$.

Compared to the simplified model, the only extra quantity required to extract the phase is the factor $\gamma=2 \alpha \frac{\omega}{c} n^{\prime} \cos \theta$, which is the index of refraction $n^{\prime}$ of the material composing the top layer and the MFP $\alpha$. The first one can be obtained from tabulated values, and the second one can be measured independently or considered as an adjustable parameter during the phase reconstruction.
F1:1

F1:2

$\mathrm{F} 1: 3$

$\mathrm{F} 1: 4$

F1:5

F1:6

$\mathrm{F} 1: 7$

$\mathrm{F} 1: 8$ 
We now illustrate this phase-extraction procedure experimentally with two periodic $\mathrm{Cr} / \mathrm{Sc}$ multilayer structures similar to the one described in Fig. 1. Both stacks (20 and 50 periods) are capped by $\sim 2 \mathrm{~nm}$ of silicon. The multilayers were chosen to obtain a Bragg's peak around $364 \mathrm{eV}$ at $45^{\circ}$ incidence for $s$ polarization. Both stacks were deposited on a silicon substrate using magnetron sputtering. The deposition parameters for the $\mathrm{Cr} / \mathrm{Sc}$ multilayer are given in [12].

Metrology has been performed using $\mathrm{Cu} \mathrm{K} \alpha$ grazing incidence $\mathrm{x}$-ray reflectometry measurements. Fitting of the experimental data gives $\sim 1.2 \mathrm{~nm}$ for $\mathrm{Cr}, \sim 1.22 \mathrm{~nm}$ for Sc, $\sim 1.97 \mathrm{~nm}$ for $\mathrm{Si}$, and $\sim 1.41 \mathrm{~nm}$ for $\mathrm{SiO}_{2}$. The interfacial roughness is estimated at $\sim 0.4 \mathrm{~nm}$ for all of the interfaces. We take into account the roughness in our simulations by using the Nevot-Croce factor.

As shown in Eq. (4), the phase extraction requires measuring both the at-wavelength reflectivity $R(\omega)$ and the normalized

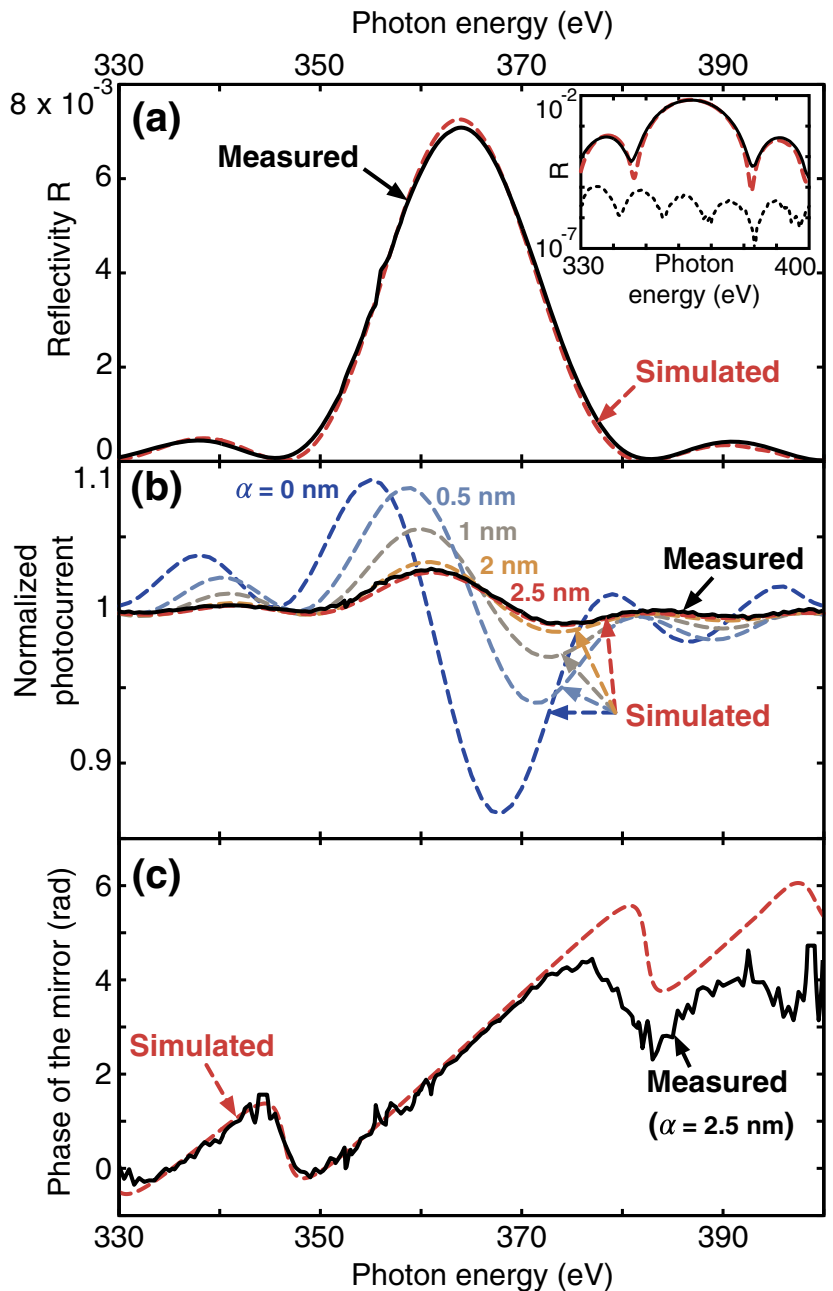
of the mirror retrieved with Eq. (4) if assuming a mean free path of $2.5 \mathrm{~nm}$.

Fig. 2. Properties of the 20 periods $\mathrm{Cr} / \mathrm{Sc}$ multilayer stack. tivity at $45^{\circ}$ incidence angle in linear (main panel) and logarithmic reflectivity at $10^{\circ}$ incidence angle. (b) Measured (continuous line) and theoretical (dashed lines) normalized photocurrents $\eta(\omega)$ for various mean free paths $\alpha$. (c) Simulated (dashed line) and experimental phase

photocurrent $\eta(\omega)$ of the sample. Such measurements were performed at the BEAR beamline at ELETTRA [13]. The experimental polarization fraction is $90 \% \mathrm{~S} ; 10 \% \mathrm{P}$, which was taken into account in simulations of the reflectivity and photocurrent in Figs. 2 and 3.

The measured reflectivities agree very well with the theoretical ones; see Fig. 2(a) in the case of the 20 periods stack and Fig. 3(a) for the 50 periods stack.

The reference photocurrent $i_{\text {ref }}$ is conveniently obtained by measuring the photocurrent of the same sample set at an incidence angle where the Bragg's law is not fulfilled. In the present case, for an incidence angle of $10^{\circ}$, the reflectivity becomes 10 to $10^{4}$ times smaller than at $45^{\circ}$, as shown in the inset in Fig. 2(a) for the 20 periods $\mathrm{Cr} / \mathrm{Sc}$ stack. Finally, the normalized photocurrent $\eta$ is defined as $i / i_{\text {ref }} \cdot \cos \left(45^{\circ}\right) / \cos \left(10^{\circ}\right)$, where the extra factor accounts for the variation of the interaction volume with the incidence angle [6]. The experimental normalized photocurrents $\eta$ are depicted in Figs. 2(b) and 3(b). Interestingly, when compared to the photocurrent predicted by Eq. (2) for a zero MFP, the measured signal exhibits the

174 175 176 177 178 179 180 181 182 183 184 185 186 187 188 189 190 191 192 193

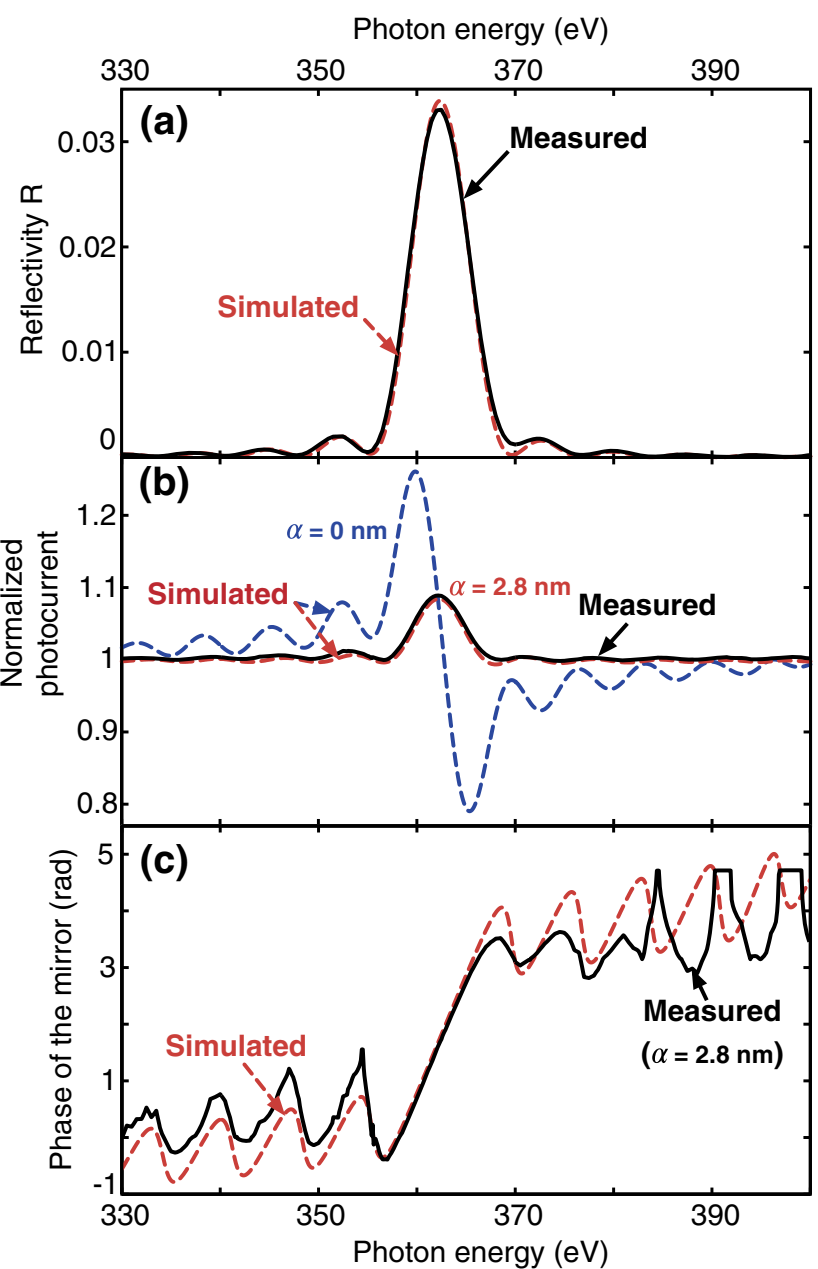

Fig. 3. Experimental (continuous lines) and theoretical (dashed lines) (a) reflectivity, (b) normalized photocurrent, and (c) phase of the 50 periods $\mathrm{Cr} / \mathrm{Sc}$ multilayer stack at $45^{\circ}$ incidence angle. In (b), the normalized photocurrent $\eta(\omega)$ is simulated for $\alpha=0$ and $2.8 \mathrm{~nm}$. In (c), an experimental mean free path of $2.8 \mathrm{~nm}$ is assumed for the phase reconstruction.
F3:1 F3:2 F3:3 F3:4 F3:5 F3:6 
same attenuation and phase shift as expected from our model. We now use the new Eq. (3) to simulate the photocurrent for various MFP. The value of $\alpha$ that best fits the experimental curve lies in the range $2.5-2.8 \mathrm{~nm}$ for both multilayer stacks. In that case, the simulated normalized photocurrents are barely distinguishable from the measured curves. Such MFP values in a silicon oxide layer are consistent with those found in literature $[14,15]$.

The phase of the mirror is deduced from Eq. (4) using the measured $R$ and $\eta$ and the value of $\alpha$ mentioned above. The phase obtained for each mirror is reported in Figs. 2(c) and $3(\mathrm{c})$. The characteristic phase behavior of a periodic stack is retrieved, that is, a linear phase across the Bragg's peak surrounded by Kissing fringes.

We believe that these results constitute a proof of principle of the phase measurement of multilayers in the soft x-ray range, and there is certainly room for improvements. Specifically, it can be seen that the noise level on the phase is rather high, especially in the case of the 20 periods stack. This is mainly due to the level of photoelectrons measured by the picoammeter. This could be improved by increasing the integration time of the photocurrent. However, all things being kept equal, we have observed in practice that the photocurrent starts decreasing slowly after illuminating the sample for more than 10 min with the synchrotron beam [16]. Although the origin of this phenomenon remains unclear, we assume a surface modification under irradiation. In the present case, this limited the integration time to $1 \mathrm{~s}$ per photon energy value.

To conclude, we performed what we think is the first phase measurement of a multilayer mirror in the soft $\mathrm{x}$ rays. To do so, we used the photocurrent technique and developed a new model accounting for the mean free path of the electrons, which becomes nonnegligible at these energies. This approach has been validated experimentally by using two $\mathrm{Cr} / \mathrm{Sc}$ periodic stacks. These results pave the way toward the development of phase-controlled mirrors at much higher energies such as chirped mirrors for attoscience and polarizing mirrors and could also be of benefit to the metrology of refraction indices in this range.
Funding. Agence Nationale de la Recherche (L' Agence 4233 Nationale de la Recherche) (ANR3113EQPX30005).

\section{REFERENCES}

1. A. Wonisch, T. Westerwalbesloh, W. Hachmann, N. Kabachnik, U. Kleineberg, and U. Heinzmann, Thin Solid Films 464-465, 473 (2004).

2. C. Bourassin-Bouchet, Z. Diveki, S. de Rossi, E. English, E. Meltchakov, O. Gobert, D. Guénot, B. Carré, F. Delmotte, P. Salières, and T. Ruchon, Opt. Express 19, 3809 (2011).

3. M. Hofstetter, M. Schultze, M. Fiess, B. Dennhardt, A. Guggenmos, J. Gagnon, V. S. Yakovlev, E. Goulielmakis, R. Kienberger, E. M. Gullikson, F. Krausz, and U. Kleineberg, Opt. Express 19, 1767 (2011).

4. S. V. Pepper, J. Opt. Soc. Am. 60, 805 (1970).

5. A. Aquila, F. Salmassi, and E. Gullikson, Opt. Lett. 33, 455 (2008).

6. M. Suman, G. Monaco, M. G. Pelizzo, D. L. Windt, and P. Nicolosi, Opt. Express 17, 7922 (2009).

7. C. Bourassin-Bouchet, S. de Rossi, J. Wang, E. Meltchakov, A. Giglia, N. Mahne, S. Nannarone, and F. Delmotte, New J. Phys. 14, 023040 (2012).

8. R. A. Loch, A. Dubrouil, R. Sobierajski, D. Descamps, B. Fabre, P. Lidon, R. W. E. van de Kruijs, F. Boekhout, E. Gullikson, J. Gaudin, E. Louis, F. Bijkerk, E. Mével, S. Petit, E. Constant, and Y. Mairesse, Opt. Lett. 36, 3386 (2011).

9. T. Popmintchev, M.-C. Chen, P. Arpin, M. M. Murnane, and H. C. Kapteyn, Nat. Photonics 4, 822 (2010).

10. T. Ejima, Jpn. J. Appl. Phys., Part 1 42, 6459 (2003).

11. M. P. Seah and W. A. Dench, Surf. Interface Anal. 1, 2 (1979).

12. F. Bridou, F. Delmotte, P. Troussel, and B. Villette, Nucl. Instrum. Methods Phys. Res. A 680, 69 (2012).

13. S. Nannarone, F. Borgatti, A. DeLuisa, B. P. Doyle, G. C. Gazzadi, A. Giglia, P. Finetti, N. Mahne, L. Pasquali, M. Pedio, G. Selvaggi, G. Naletto, M. G. Pelizzo, and G. Tondello, Nucl. Instrum. Methods Phys. Res. A 705, 450 (2004).

14. J. C. Ashley and V. E. Anderson, J. Electron Spectrosc. Relat. Phenom. 24, 127 (1981).

15. M. Kasrai, W. N. Lennard, R. W. Brunner, G. M. Bancroft, J. A. Bardwell, and K. H. Tan, Appl. Surf. Sci. 99, 303 (1996).

16. F. Delmotte, C. Bourassin-Bouchet, S. de Rossi, E. Meltchakov, A. Giglia, and S. Nannarone, Proc. SPIE 9207, 92070 (2014). 


\section{Queries}

1. AU: Please note that references are cited out of order. Hence references are renumbered and citations are modified accordingly.

2. AU: Please define CXRO in the paragraph beginning, "In order to validate this model

3. AU: Please define BEAR in paragraph beginning, "As shown in Eq. (4), the phase extraction requires ..................."

4. The funding information for this article has been generated using the information you provided to OSA at the time of article submission. Please check it carefully. If any information needs to be corrected or added, please provide the full name of the funding organization/institution as provided in the FundRef Registry (http://www.crossref.org/fundref/fundref_registry.html). 